\title{
Analyticity of Effective Coupling and Propagators in Massless Models of Quantum Field Theory
}

\author{
Reinhard Oehme ${ }^{1}$ and Wolfhart Zimmermann ${ }^{2 \star}$ \\ 1 The Enrico Fermi Institute and the Department of Physics, The University of Chicago, Chicago, \\ IL 60637, USA \\ 2 The Institute for Advanced Study, Princeton, NJ 08540, USA, and Rockefeller University, New \\ York, NY 10021, USA
}

\begin{abstract}
For massless models of quantum field theory, some general theorems are proved concerning the analytic continuation of the renormalization group functions as well as the effective coupling and the propagators. Starting points are analytic properties of the effective coupling and the propagators in the momentum variable $k^{2}$, which can be converted into analyticity of $\beta$ - and $\gamma$-functions in the coupling parameter $\lambda$. It is shown that the $\beta$-function can have branch point singularities related to stationary points of the effective coupling as a function of $k^{2}$. The type of these singularities of $\beta(\lambda)$ can be determined explicitly. Examples of possible physical interest are extremal values of the effective coupling at space-like points in the momentum variable, as well as complex conjugate stationary points close to the real $k^{2}$-axis. The latter may be related to the sudden transition between weak and strong coupling regimes in quantum chromodynamics. Finally, for the effective coupling and for the propagators, the analytic continuation in both variables $k^{2}$ and $\lambda$ is discussed.
\end{abstract}

\section{Introduction}

For massless models of quantum field theory, the renormalization group may be used in order to obtain analytic properties of Green's functions in the coupling parameter $\lambda .^{1}$ It is the purpose of this paper to derive some general theorems concerning the analytic continuation of the effective coupling $\Lambda$ and of the propagators in the parameter $\lambda$ and the momentum variable $k^{2}$. In particular, we show that the renormalization group function $\beta$ can have well defined branch points which are associated with stationary points of the effective coupling $\Lambda$ as a function of $k^{2}$. These branch points may be of physical interest in quantum chromodynamics. $^{2}$

\footnotetext{
* On leave from the Max-Planck-Institut für Physik und Astrophysik, D-8000 München, Federal Republic of Germany

1 For previous work on the analytic continuation in the coupling constant, see [1-5]

2 A preliminary account of this work was given in [5-7]
} 
We assume in this paper that the renormalization group transformations involve only two independent parameters; a dimensionless coupling parameter $\lambda$ and a normalization mass $\kappa^{2}$. This is the case for the Landau gauge of massless gauge theories with a single dimensionless coupling constant. Accordingly, for such models our treatment applies to gauge invariant quantities or gauge dependent quantities in the Landau gauge. Though intrinsic masses are absent, the possibility of dynamical mass generation is included.

Our starting point will be the analytic properties of the effective coupling $\Lambda$ as a function of $k^{2}$ for fixed real $\lambda \geqq 0$. We follow the approach to define the effective coupling $\Lambda$ in terms of a suitably chosen vertex function which depends only upon a single momentum variable $k^{2}$. Thus defined, the effective coupling may be regarded as a measure of the coupling strength in its dependence upon distance or momentum. ${ }^{3}$ The definition of $\Lambda$ is, of course, not unique. Except for quantum electrodynamics, there is no preferred choice of the coupling parameter. Nevertheless, it can be expected that the essential features of the effective coupling as a function of the momentum are similar for all definitions which are based on vertex functions in the Euclidean momentum region. In view of the relation to time ordered functions, the effective charge is the boundary value of an analytic function in $k^{2}$. Different constructions of $\Lambda$, which are regular analytic at any $k^{2}<0$, are related by reparametrization transformations which are nonsingular in the entire Euclidean region. For some definitions $\Lambda$ will even be analytic in the cut $k^{2}$-plane for real $\lambda \geqq 0[10]$. For the general considerations of this paper, the precise extent of the region of analyticity in $k^{2}$ will not be relevant. It is sufficient if we have regularity in some domain of a Riemann surface over the $k^{2}$-plane, which includes an appropriate interval on the negative real $k^{2}$-axis. The renormalization group function $\beta(\lambda)$ then becomes analytic in the domain of values which the effective charge $\Lambda$ assumes at its points of regularity in $k^{2}$. In this domain the function $\beta$ is regular except at stationary values of $\Lambda$ with $\partial \Lambda / \partial k^{2}=0$.

It is important to distinguish two types of singularities of the function $\beta(\lambda)$. First, there are those which are associated with poles and branch points of the effective coupling $\Lambda$ as a function of $k^{2}$, which are due to the intermediate states in the appropriate channels of the vertex function and the propagators used in the definition of $\Lambda$. Since inverse square roots of propagators are involved, there could also be contributions from zeros of propagators, unless appropriate projected propagators are used.

A second type of singularities of the function $\beta(\lambda)$ is caused by zeros of $\partial \Lambda / \partial k^{2}$, which may occur anywhere in the regularity domain of $\Lambda$ as a function of $k^{2}$. At these stationary points of $\Lambda$, the $\beta$-function has branch points whose general form can be determined to be $[6,7]$

$$
\begin{gathered}
\beta=\left(\lambda-\lambda^{\prime}\right)^{1-1 / n}\left(B_{0}+\left(\lambda-\lambda^{\prime}\right)^{1 / n} B_{1}+\ldots+\left(\lambda-\lambda^{\prime}\right)^{1-1 / n} B_{n-1}\right), \\
n=2,3, \ldots
\end{gathered}
$$

with coefficients $B_{i}$ regular at $\lambda^{\prime}$. Here $\lambda^{\prime}$ is the value of $A$ at a stationary point $k^{2} \neq 0$ where $A$ is regular analytic, $n$ is the order of the first nonvanishing derivative of $\Lambda$, and $\beta$ vanishes at such a branch point with $\beta^{-1}$ being integrable.

3 For this approach of defining the effective coupling see, for example $[8,9]$ 
Some applications of singularities associated with stationary values of $\Lambda$ have been discussed previously. For real Euclidean $k^{2}$, stationary values of the effective coupling are completely consistent with the concept of the renormalization group [6]. ${ }^{4}$ For an extremal value $\lambda^{\prime}$ of $\Lambda$ the function $\beta(\lambda)$ passes through the real axis at $\lambda^{\prime}$ with the positive and negative branch on one side of the vertical tangent $\lambda=\lambda^{\prime}$. Then the field quantities become multi-valued functions of the coupling constant. Another interesting example is the case where, at a point $k^{2}<0$, the first two derivatives of $\Lambda$ vanish, but not the third one. At the corresponding stationary value, $\beta$ does not change sign, but vanishes with a cusp-like behavior. Finally, we mention an application of complex branch points of $\beta$. It has been suggested that the indication of an abrupt transition between real and strong coupling regions, $a$ priori found in lattice gauge theories, may be connected to complex conjugate branch points of $\beta$ close to the real axis $[13,14,7]$. The possibility that such singularities may be due to complex stationary values of the effective coupling was discussed in [7].

For positive $\lambda$, the propagator of an elementary or composite field is regular analytic in the cut $k^{2}$-plane with the possibility of a continuation across the cut. For propagators which transform multiplicatively under the renormalization group, analyticity in momentum space implies analyticity of the anomalous dimension $\gamma$ in the coupling constant.

With analyticity established for the $\beta$ - and $\gamma$-functions, an analytic continuation of $\Lambda$ and of the propagators in both variables $\lambda$ and $k^{2}$ may be accomplished. We find fixed singularities in $\lambda$ and $k^{2}$, as well as singular surfaces in both variables. Fixed singularities in $k^{2}$ can only occur at the origin or at infinity. Fixed singularities in $\lambda$ may be located at the singularities of $\beta^{-1}$ (and/or $\gamma \beta^{-1}$ for propagators). The singular surfaces have the simple structure given in [5], which is an extension of the familiar $\lambda$-dependence for observable masses to complex values of $\lambda$ and $k^{2}$.

Analyticity in the coupling parameter may be severely restricted by singularities in momentum space $[4,5,15]$. In massless quantum chromodynamics, Khuri has proved that propagators with thresholds extending to infinity cannot be continued in any coupling parameter beyond a certain domain where boundaries are tangential to the real axis at the origin. ${ }^{5}$ This limitation is due to the infinite sequence of singular surfaces originating in the threshold singularities of Minkowski space.

The methods of the present paper apply as well to definitions of the effective coupling which are based on an explicitly given $\beta$-function. Starting from a given $\beta$, the effective coupling is defined by

$$
\frac{k^{2}}{\kappa^{2}}=\exp \left(\int_{\lambda}^{\Lambda} d z \beta^{-1}\right)
$$

in an appropriate range of the variables $k^{2}, \Lambda$. This relation can be continued to complex values of $\lambda, k^{2}$ and $\Lambda$ by deforming the path of integration within the

4 Examples of stationary points for the effective coupling have been found in the $1 / N$ limit of a model [11] and in an approximative treatment of QCD for a certain range of the $\theta$-parameter [12] 5 By a different method the same limitation was obtained in [16] for a large class of coupling reparametrizations 
regularity domain of $\beta^{-1}$. Thus the analytic continuation of $\lambda$ is obtained in both variables $k^{2}$ and $\lambda$.

In Sect. 2, the concept of the renormalization group in the general framework of quantum field theory is reviewed as far as needed for the purpose of this paper. The analytic properties of the renormalization group functions $\beta$ and $\gamma$ are derived in Sect. 3 from the regularity domain of the effective coupling and the propagators in momentum space. The continuation in both variables, the coupling parameter and the momentum variable, is discussed in Sect. 4.

\section{Renormalization Group}

Massless models of quantum field theory are considered which involve a single dimensionless coupling constant $\lambda$ and a Euclidean normalization mass $\kappa$. Let $\phi_{1}, \ldots, \phi_{n}$ denote the basic field operators of the model (Lorentz and internal symmetry indices suppressed). The renormalization group is defined as the group of all transformations

$$
\phi_{r} \rightarrow Z_{r}^{1 / 2} \phi_{r}, \quad Z_{r}>0
$$

which change the normalization of the fields by positive factors. It is assumed that a two-parameter family of normalized field operators

$$
\phi_{r}=\phi_{r}\left(x, \lambda, k^{2}\right)
$$

exists which is uniquely determined by suitable normalization conditions for coupling constants from a sufficiently small region

$$
0 \leqq \lambda \leqq \eta
$$

and a normalization mass

$$
\kappa^{2}<0
$$

Parameter values $\lambda, \kappa^{2}$ and $\lambda^{\prime}, \kappa^{\prime 2}$ are called equivalent:

$$
\lambda, \kappa^{2} \sim \lambda^{\prime}, \kappa^{\prime 2},
$$

if the corresponding field operators are related by an equivalence transformation.

The effective coupling is defined by a dimensionless quantity ${ }^{6}$

$$
\begin{aligned}
\Lambda & =\Lambda\left(k^{2}, \lambda, \kappa^{2}\right) \\
& =\Lambda(u, \lambda), \quad u=k^{2} / \kappa^{2}
\end{aligned}
$$

depending on the square of a four vector $k$ which is invariant under the renormalization group:

$$
\Lambda\left(k^{2}, \lambda, \kappa^{2}\right)=\Lambda\left(k^{2}, \lambda^{\prime}, \kappa^{\prime 2}\right)
$$

6 For gauge theories, $\lambda$ may be taken as square of the conventional coupling constant with $A$ denoting the square of the effective coupling 
and satisfies the condition

$$
\Lambda\left(k^{2}, \lambda, \kappa^{2}\right)=\lambda \quad \text { at } k^{2}=\kappa^{2} .
$$

From (2.7) and (2.8) it follows that equivalent pairs (2.5) of parameter values are related by

$$
\lambda^{\prime}=\Lambda\left(\kappa^{\prime 2}, \lambda, \kappa^{2}\right)=\Lambda\left(\frac{\kappa^{\prime 2}}{\kappa^{2}}, \lambda\right) .
$$

Equations (2.6) and (2.7) imply the differential equation

$$
u \frac{\partial \Lambda}{\partial u}=\beta(\lambda) \frac{\partial \Lambda}{\partial \lambda} .
$$

Only such models will be considered where

$$
\beta(0)=0, \text { but } \beta(\lambda) \neq 0 \text { for small } \lambda>0 .
$$

Then one has the alternative that

$$
\left.\begin{array}{ll}
\text { either } & \beta(\lambda)>0 \\
\text { or } & \beta(\lambda)<0
\end{array}\right\} \text { for small } \lambda>0 \text {. }
$$

With (2.8) the differential equation (2.10) can be solved by

$$
u=\exp \left(\int_{\lambda}^{A} d z \beta^{-1}\right)
$$

for

or

$$
\begin{array}{lll}
0 \leqq \lambda \leqq \eta, & 0 \leqq u \leqq c, & \beta(\lambda)>0 \\
0 \leqq \lambda \leqq \eta, & u \geqq c, & \beta(\lambda)<0
\end{array}
$$

with suitable bounds $\eta$ and $c$ such that in particular

$$
\frac{\partial \Lambda}{\partial u} \neq 0, \quad \beta(\lambda) \neq 0
$$

in the intervals (2.13). With (2.12) the relation (2.9) between equivalent pairs of parameter values may be written as

$$
\frac{\kappa^{\prime 2}}{\kappa^{2}}=\exp \left(\int_{\lambda}^{\lambda^{\prime}} d z \beta^{-1}\right) .
$$

For the Callan-Symanzik function $\beta$, the relation

$$
\beta(\Lambda)=u \frac{\partial \Lambda}{\partial u}
$$

can be derived from (2.7) and (2.9) provided $0 \leqq \Lambda \leqq \eta$. On the right hand side of this relation $u$ and $\partial \Lambda / \partial u$ are to be expressed by the variables $\Lambda$ and $\lambda$. The resulting expression is independent of $\lambda$. In the discussion of singularities of $\beta$ and $\Lambda$, as well as stationary points of $\Lambda$, we must remember that these positions can generally be changed by reparametrization transformations. If singular transfor- 
mations are admitted, we can, of course, remove or generate singularities, for example in $\beta$, but at the cost of compensating changes in $\Lambda$ which then will no longer have a direct relationship to a vertex function and its role as a measure of the coupling strength as a function of momentum or distance. Nevertheless, singular reparametrizations may be of relevance within the framework of the exact theory or nonperturbative approximation methods [4, 15, 17-19].

Apart from the basic fields $\phi_{r}$ occurring in the Lagrangian, also composite field operators $C$ will be considered which transform multiplicatively under the equivalence transformations (2.1),

$$
C \rightarrow Z_{C}^{1 / 2} C .
$$

Let $G\left(k^{2}\right)$ denote a structure function of a basic field $\phi_{r}$ or a composite operator with multiplicative transformation law (2.16). Then

$$
G\left(k^{2}, \lambda^{\prime}, \kappa^{\prime 2}\right)=Z G\left(k^{2}, \lambda, \kappa^{2}\right)
$$

with $Z=Z_{r}$ or $Z=Z_{C}$. This implies the differential equation

$$
\begin{aligned}
\kappa^{2} \frac{\partial G}{\partial \kappa^{2}}+\beta \frac{\partial G}{\partial \lambda}+\gamma G & =0, \\
\gamma & =\gamma(\lambda),
\end{aligned}
$$

with the anomalous dimension $\gamma$ depending on the field operator considered. Multiplying by a suitable power of $k^{2}$, we form the dimensionless quantity

$$
R(u, \lambda)=\left(k^{2}\right)^{a} G, \quad u=\frac{k^{2}}{\kappa^{2}},
$$

satisfying (2.18) in the form

$$
u \frac{\partial R}{\partial u}=\beta \frac{\partial R}{\partial \lambda}+\gamma R
$$

Changing the normalization of the field operator by a $\lambda$-dependent factor, it can always be arranged that

$$
R(u, \lambda)=1 \quad \text { at } u=1
$$

In the work that follows, normalized structure functions $R$ will be considered which satisfy (2.21). With (2.21), the differential equation (2.20) can be solved to yield

$$
R=\exp \left(\int_{\lambda}^{\Lambda} d x \gamma \beta^{-1}\right),
$$

by using $\Lambda$ and $\lambda$ as independent variables. The variables $\lambda$ and $u$ should be restricted by suitable bounds as in (2.13). With the normalization condition (2.21), the factor $Z$ of the transformation law (2.17) can be determined as

$$
Z=\exp \left(\int_{\lambda^{\prime}}^{\lambda} d x \gamma \beta^{-1}\right) .
$$


It is sometimes convenient to replace a propagator

$$
\left\langle T \phi_{r a}(x) \phi_{r b}(y)\right\rangle
$$

by $[20-22]$

$$
\left\langle T \phi_{r a}^{+}(x) \phi_{r b}^{+}(y)\right\rangle=\left\langle T \phi_{r a}(x) p_{r} \phi_{r b}(y)\right\rangle
$$

where $p_{r}$ is the projection on a subspace $H_{r}^{+}$of positive definite metric of the space $H_{r}$ of all states with

$$
\left(\Omega, \phi_{r a}(x) \Phi\right) \neq 0
$$

The corresponding structure functions are denoted by $G^{+}$. The quantity

$$
R^{+}=\left(p^{2}\right)^{a} G^{+}
$$

is dimensionless and depends on $u$ and $\lambda$ only,

$$
R^{+}=R^{+}(u, \lambda), \quad u=k^{2} / \kappa^{2} .
$$

If the definition of $H_{r}^{+}$does not involve additional mass parameters, it satisfies the differential equation

$$
u \frac{\partial R^{+}}{\partial u}=\beta \frac{\partial R^{+}}{\partial \lambda}+\gamma R^{+}
$$

with the same coefficients $\beta$ and $\gamma$.

The structure functions $G$ of the propagators are analytic functions in the cut $k^{2}$-plane. The effective coupling is also expected to be analytic in $k^{2}$. For suitable definitions of the effective coupling, it can be shown that it is analytic in the cut $k^{2}$-plane [6]. $G$ and $\Lambda$ may possibly be continued across the cut as analytic functions on a Riemann surface. The renormalization group transformations (2.7) and (2.17) can be continued to any value of $k^{2}$ where $A$ or $G$ are regular. In terms of dimensionless variables, these transformations take the form

$$
\begin{array}{r}
\Lambda(u, \lambda)=\Lambda\left(\frac{\kappa^{2}}{\kappa^{\prime 2}} u, \lambda^{\prime}\right), \\
R(u, \lambda)=Z R\left(\frac{\kappa^{2}}{\kappa^{\prime 2}} u, \lambda^{\prime}\right) .
\end{array}
$$

Inserting (2.14) and (2.23), one finds the functional relations

$$
\begin{gathered}
\Lambda(u, \lambda)=\Lambda\left(\exp \left(\int_{\lambda^{\prime}}^{\lambda} d z \beta^{-1}\right) u, \lambda^{\prime}\right), \\
R(u, \lambda)=\exp \left(\int_{\lambda}^{\lambda^{\prime}} d z \gamma \beta^{-1}\right) R\left(\exp \left(\int_{\lambda^{\prime}}^{\lambda} d z \beta^{-1}\right) u, \lambda^{\prime}\right),
\end{gathered}
$$

valid wherever $\Lambda$ and $R$ are regular analytic in $u$.

We will now show that the position

$$
\mu^{2}=\mu^{2}\left(\lambda, \kappa^{2}\right)
$$


of a singularity of $G$ or $\Lambda$ on the Riemann surface depends on the coupling parameter like [5]

$$
\mu^{2}=\mu_{0}^{2} \exp \left(\int_{\lambda}^{\lambda_{0}} d z \beta^{-1}\right), \quad 0 \leqq \lambda \leqq \eta, 0 \leqq \lambda_{0} \leqq \eta .
$$

This law is well known to hold for observable mass parameters for any renormalizable massless model with a single coupling constant $\lambda$. For the proof we first observe that the location of a singularity of $G$ or $\Lambda$ cannot change under an equivalence transformation (2.1) which only multiplies the function by a finite number [see (2.7) and (2.17)]. Therefore,

$$
\begin{aligned}
\mu^{2}\left(\lambda^{\prime}, \kappa^{\prime 2}\right) & =\mu^{2}\left(\lambda, \kappa^{2}\right), \\
\lambda^{\prime}, \kappa^{\prime 2} & \sim \lambda, \kappa^{2} .
\end{aligned}
$$

The same relation holds for the observables of the system since, by definition, an observable does not depend on the normalization of the fields. The invariance relation (2.33) implies

$$
\kappa^{2} \frac{\partial \mu^{2}}{\partial \kappa^{2}}+\beta \frac{\partial \mu^{2}}{\partial \lambda}=0
$$

For dimensional reasons

$$
\mu^{2}\left(\lambda, \kappa^{2}\right)=\kappa^{2} f(\lambda)
$$

Therefore (2.34) becomes

$$
\begin{gathered}
\beta \frac{\partial f}{\partial \lambda}+f=0, \\
f=f_{0} \exp \left(\int_{\lambda}^{\lambda_{0}} d z \beta^{-1}\right) .
\end{gathered}
$$

Thus (2.32) holds for any singularity of the effective coupling or a propagator. According to the derivation, it is also fulfilled for those singularities at which the function assumes a finite value.

Particularly interesting is the case of the lowest singularity $m^{2}$ on the axis $k^{2} \geqq 0$. Either $m$ vanishes identically or depends on the coupling parameter according to (2.32). For a propagator, $m$ is the lowest mass value of the modes associated with the corresponding fields. In case of the Fermi propagators of quantum chromodynamics, these values may be used for defining the quark masses [22].

The location of a zero of $G$ or $A$ also stays invariant under equivalence transformations. Therefore a $\lambda$-dependence similar to (2.32) follows for the position of a zero on the Riemann surface over the $k^{2}$-plane. 


\section{Analyticity of $\beta$ and $\gamma$}

In this section it will be shown how analyticity of the effective coupling and the propagators in the momentum variable alone can be converted to analyticity of the $\beta$ - and the $\gamma$-functions in the coupling parameter.

We introduce a domain $\mathscr{D}$ as the set of all values which

$$
\Lambda=\Lambda\left(k^{2}, \lambda, \kappa^{2}\right)
$$

assumes at regular points $k^{2}$. In general, $\mathscr{D}$ will be a domain on a Riemann surface over the $\Lambda$-plane. Here $\mathscr{D}$ does not depend on $\kappa^{2}$ since $\Lambda$ is a function of $k^{2} / \kappa^{2}$ and $\Lambda$ only. Due to the invariance relation (2.7), $\mathscr{D}$ does not depend on $\lambda$ either.

For any value of $\Lambda$ within $0 \leqq \Lambda \leqq \eta$, we have [Eq. (2.14)]

$$
\beta(\Lambda)=u \frac{\partial \Lambda}{\partial u} .
$$

This relation may be continued to complex values of $\Lambda$ in $\mathscr{D}$. It implies that $\beta$ is an analytic function, regular in $\mathscr{D}$ except at points where $\Lambda=\Lambda(u, \lambda)$ cannot be inverted with respect to $u$. Hence, within the domain $\mathscr{D}$, the function $\beta$ as given by (3.1) can only become singular if

$$
\frac{\partial \Lambda}{\partial u}=0
$$

Because of (3.1), $\beta$ always vanishes at a singularity in $\mathscr{D}$.

The effective coupling $\Lambda$ is usually defined as a real function of Euclidean momenta $k^{2}<0$ for $0 \leqq \lambda \leqq \eta$. For some definitions, $\Lambda$ is real only in a domain $\mathscr{E}$ which need not be the full Euclidean region. By definition, $\beta$ becomes real in the region $\mathscr{F}$ of values which $\Lambda$ assumes in $\mathscr{E}$. For $\lambda \in \mathscr{F}$ the analytic functions $\beta(\lambda)$ and $\bar{\beta}(\lambda)=\beta^{*}\left(\lambda^{*}\right)$ coincide, hence represent the same analytic functions. As a consequence, complex singularities of $\beta$ appear in pairs at complex conjugate locations.

We now determine the analytic properties of $\beta$ near a zero of $\partial \Lambda / \partial u$ where $\Lambda$ itself is regular in $u$. It will first be shown that the value $\lambda^{\prime}$ of $\Lambda$ at such a point does not depend on the coupling parameter. The defining equations are

$$
\lambda^{\prime}\left(\lambda_{0}\right)=\Lambda\left(u^{\prime}, \lambda_{0}\right), \quad \frac{\partial \Lambda}{\partial u}\left(u^{\prime}, \lambda_{0}\right)=0 .
$$

For two different values $\lambda_{1}, \lambda_{2}$ of the coupling constant, the effective coupling $\Lambda$ is related by $(2.29)$

$$
\begin{aligned}
\Lambda\left(u, \lambda_{2}\right) & =\Lambda\left(v, \lambda_{1}\right), \\
v & =\exp \left(\int_{\lambda_{1}}^{\lambda_{2}} d z \beta^{-1}\right) u .
\end{aligned}
$$

According to this

$$
\frac{\partial \Lambda}{\partial u}\left(u, \lambda_{1}\right)=0 \quad \text { implies } \frac{\partial \Lambda}{\partial u}\left(u, \lambda_{2}\right)=0
$$


with constant value of $\Lambda$. Hence

$$
\frac{\partial \lambda^{\prime}}{\partial \lambda_{0}}=0
$$

follows.

The value $\lambda_{0}$ of the coupling constant will be held fixed in the work that follows. If $\Lambda$ is regular at $u=u^{\prime}$ with vanishing derivative, the difference $\Lambda-\lambda^{\prime}$ must be of the form

$$
\Lambda\left(u, \lambda_{0}\right)-\lambda^{\prime}=\left(u-u^{\prime}\right)^{n} f\left(u, \lambda_{0}\right) \quad n=2,3, \ldots
$$

with

$$
f^{\prime}=f\left(u^{\prime}, \lambda_{0}\right) \neq 0 .
$$

The analytic properties of $\beta(\Lambda)$ near $\Lambda=\lambda^{\prime}$ are conveniently described in terms of a variable $z$ introduced by the analytic function

$$
\begin{aligned}
z=z\left(u, \lambda_{0}\right) & =\left(u-u^{\prime}\right) f^{1 / n}\left(u, \lambda_{0}\right) \\
& =\left(\Lambda\left(u, \lambda_{0}\right)-\lambda^{\prime}\right)^{1 / n} .
\end{aligned}
$$

The function $z$ is regular and vanishes at $u=u^{\prime}$. Its derivative does not vanish at $u^{\prime}$ :

$$
\frac{\partial z}{\partial u}\left(u^{\prime}, \lambda_{0}\right)=f^{1 / n}\left(u^{\prime}, \lambda_{0}\right) \neq 0
$$

Hence

$$
z=z\left(u, \lambda_{0}\right)
$$

may be inverted by the analytic function

$$
u=u\left(z, \lambda_{0}\right) .
$$

Since $u$ is regular at $z=0$, it may be expanded with respect to powers of $z$ :

$$
u=u^{\prime}+\left(f^{\prime}\right)^{-1 / n} z+a_{2} z^{2}+\ldots
$$

In the neighborhood of $u=u^{\prime}$, the right hand side of (3.1) will now be expressed by the variable $z$. It is

$$
u \frac{\partial \Lambda}{\partial u}=n \frac{u}{\partial u / \partial z} z^{n-1} .
$$

This is regular at $z=0$ since

$$
\frac{\partial u}{\partial z}=\left(f^{\prime}\right)^{-1 / n} \neq 0 \quad \text { at } \quad u=u^{\prime}
$$

The leading power of $z$ depends on whether or not $u^{\prime} \neq 0$. We have either

$$
\beta(\Lambda)=z^{n-1}\left(b_{0}+b_{1} z+b_{2} z^{2}+\ldots\right)
$$

with $b_{0}=n u^{\prime} f^{1 / n} \neq 0$ if $u^{\prime} \neq 0$, or

$$
\beta(\Lambda)=z^{n}\left(b_{1}+b_{2} z+\ldots\right)
$$


with $b_{1}=n=2,3, \ldots$ if $u^{\prime}=0$. In these expansions we collect terms of equal power modulo $n$

$$
\begin{aligned}
& \beta(\Lambda)=z^{n-1}\left(B_{0}+z B_{1}+\ldots+z^{n-1} B_{n-1}\right), \\
& \beta(\Lambda)=z^{n}\left(B_{0}+z B_{1}+\ldots+z^{n-1} B_{n-1}\right) .
\end{aligned}
$$

Here the $B_{j}$ are analytic functions of

$$
z^{n}=\Lambda-\lambda^{\prime},
$$

which are regular at $z=0$ or $\Lambda=\lambda^{\prime}$. At $z=0$, the coefficient $B_{0}$ of the leading term does not vanish. Since $\beta$ and $\lambda^{\prime}$ are independent of $\lambda_{0}$, the coefficients $B_{j}$ do not depend on $\lambda_{0}$ either. Replacing $\Lambda$ by $\lambda$, we find

$$
\begin{gathered}
\beta(\lambda)=\left(\lambda-\lambda^{\prime}\right)^{1-1 / n}\left(B_{0}+\left(\lambda-\lambda^{\prime}\right)^{1 / n} B_{1}+\ldots+\left(\lambda-\lambda^{\prime}\right)^{1-1 / n} B_{n-1}\right), \\
\text { if } u^{\prime} \neq 0 \quad \text { with } \quad B_{0}\left(\lambda^{\prime}\right) \neq 0, \\
\beta(\lambda)=\left(\lambda-\lambda^{\prime}\right)\left(B_{0}+\left(\lambda-\lambda^{\prime}\right)^{1 / n} B_{1}+\ldots+\left(\lambda-\lambda^{\prime}\right)^{1-1 / n} B_{n-1}\right) \\
\text { if } u^{\prime}=0 \quad \text { with } B_{0}\left(\lambda^{\prime}\right)=n
\end{gathered}
$$

as a representation of $\beta$ near $\lambda=\lambda^{\prime}$. The coefficients

$$
B_{i}=B_{i}(\lambda)
$$

are regular at $\lambda=\lambda^{\prime}$. By (3.1) and (3.2) or (3.13) and (3.14), the $\beta$-function vanishes at a stationary value of $\Lambda$. If $u^{\prime} \neq 0$, the inverse of $\beta$ is still integrable at $\lambda^{\prime}$.

This result is of particular interest for real values of $\lambda$. If $n$ is even, $\Lambda$ has an extremal value $\lambda^{\prime}$ at the point $u=u^{\prime}$. It has been pointed out in [6] that extremal values of the effective coupling are compatible with the concept of the renormalization group. We will now discuss the analytic behavior of $\beta$ near an extremal value of $\Lambda$.

We first consider $\beta$ for real arguments corresponding to values $u>u^{\prime}$ or $u<u^{\prime}$ near the location $u^{\prime} \neq 0$ of a maximum $\lambda^{\prime}$. It is then convenient to draw the cut of the branch point from $\lambda^{\prime}$ to the right along $\lambda \geqq \lambda^{\prime}$. Here $f^{\prime}=f\left(u^{\prime}, \lambda_{0}\right)$ is negative because of (3.4) and we may choose the positive root for $(-f)^{1 / n}$ in a neighborhood of $u=u^{\prime}$. With this convention, $(-1)^{1 / n} z$ and $u-u^{\prime}$ have equal signs by (3.6) so that

$$
\begin{array}{lll}
(-1)^{1 / n} z=\left(\lambda^{\prime}-\Lambda\right)^{1 / n}>0 & \text { if } \quad u>u^{\prime}, \\
(-1)^{1 / n} z=-\left(\lambda^{\prime}-\Lambda\right)^{1 / n}<0 & \text { if } \quad u<u^{\prime}
\end{array}
$$

for $u$ sufficiently close to $u^{\prime}$. Thus the representation of $\beta$ near a maximum of the effective coupling at a point $u^{\prime} \neq 0$ becomes $^{7}$

$$
\begin{aligned}
\beta(\lambda)= \pm\left(\lambda^{\prime}-\lambda\right)^{1-1 / n}\left(B_{0} \pm\right. & \left(\lambda^{\prime}-\lambda\right)^{1 / n} B_{1} \\
& \left.+\left(\lambda^{\prime}-\lambda\right)^{2 / n} B_{2} \pm \ldots \pm\left(\lambda^{\prime}-\lambda\right)^{1-1 / n} B_{n-1}\right)
\end{aligned}
$$

with the upper (lower) sign holding for the branch corresponding to $u>u^{\prime}$ (or $u<u^{\prime}$ respectively) in a sufficiently small neighborhood of $u^{\prime}$. For both branches we have

7 A qualitative picture of the $\lambda$-dependence of $\beta$ in case of a single maximum of $\Lambda$ was given in [6, Fig. 1] 
$\lambda \leqq \lambda^{\prime}$. The coefficients $B_{i}$ are real functions near $u^{\prime}$ with $B_{0} \neq 0$. In case of a minimum, the representation (3.15) holds after replacing $\lambda^{\prime}-\lambda$ by $\lambda-\lambda^{\prime}$.

It is interesting to study the analytic properties of $\beta$ near such a branch point. If $u$ is continued from $u^{\prime}-\varepsilon$ to $u^{\prime}+\varepsilon(\varepsilon>0)$ along a complex path in the upper or lower half plane, the effective coupling $\Lambda$ will make $n / 2$ full turns on the Riemann surface around the branch point at $\lambda^{\prime}$. Accordingly the two branches (3.15) of $\beta$ are connected by continuing $n / 2$-times around $\lambda=\lambda^{\prime}$ on the Riemann surface of $\left(\lambda-\lambda^{\prime}\right)^{1 / n}$.

If $n$ is odd, the effective coupling is stationary but does not have an extremum. We discuss this case first for a real neighborhood of a stationary point at $u=u^{\prime} \neq 0$. Then the function $f$ defined by (3.4) is real. Choosing the real root $f^{1 / n}$ in (3.6), the representation (3.13) of $\beta$ holds in a real neighborhood of $\lambda^{\prime}$ with real roots $\left(\lambda-\lambda^{\prime}\right)^{1 / n}$. Analytic continuation of $\beta$ from values $\lambda<\lambda^{\prime}$ to $\lambda>\lambda^{\prime}$ must be performed in the Riemann surface of the function $\left(\lambda-\lambda^{\prime}\right)^{1 / n}$ by taking $n / 2$ turns around $\lambda=\lambda^{\prime}$.

We briefly indicate the modifications if $\Lambda$ has a stationary value at $u=0$. For an extremum ( $u$ even) the factor $\pm\left|\lambda-\lambda^{\prime}\right|^{-1 / n}$ in Eq. (3.15) is replaced by $\lambda-\lambda^{\prime}$. Both branches of $\beta$ form a cusp with the same tangent given by $B_{0}\left(\lambda^{\prime}\right)=n$. If $n$ is odd, we have (3.14) with the real root of $\left(\lambda-\lambda^{\prime}\right)$. In that case $\beta$ is passing continuously through zero at $\lambda=\lambda^{\prime}$ with tangent $B_{0}\left(\lambda^{\prime}\right)=n$.

It has been suggested in $[7,13,14]$ that complex branch point singularities of the $\beta$-function may be related to the sudden transition from a weak coupling to a strong coupling approximation. Complex stationary points of the effective coupling may provide such a pair of conjugate singularities for the $\beta$-function. In that case the representation (3.13) holds locally at each of the singularities. It is more difficult to obtain a representation valid jointly for conjugate singularities. In particular, we emphasize that conjugate stationary points on the physical sheet of $\Lambda$ may lead to conjugate singularities of $\beta$, which are located on complex conjugate sheets rather than the original physical sheet of the Riemann surface. Likewise, conjugate singularities of $\beta$ on the physical sheet need not correspond to conjugate stationary points on the physical sheet of $\Lambda$. In some simple models, the branch points may also be closely correlated with meromorphic singularities on the real $\lambda$-axis.

We conclude this section by deriving analytic properties for the anomalous dimension $\gamma(\lambda)$ of a normalized propagator. From the differential equation (2.20) and the normalization condition (2.21), the formula

$$
\gamma(\lambda)=\left.\frac{\partial R(v, \lambda)}{\partial v}\right|_{v=1}
$$

follows. We write the transformation (2.28) in the form

$$
R(v, \lambda)=Z R\left(u v, \lambda_{0}\right)
$$

with

$$
\lambda=\lambda\left(u, \lambda_{0}\right), \quad u=\frac{\kappa^{2}}{\kappa_{0}^{2}} ;
$$

$\lambda_{0}$ denotes a positive value below $\eta$, with $\eta$ chosen such that not only $\beta\left(\lambda_{0}\right) \neq 0$ but also $R\left(u, \lambda_{0}\right) \neq 0$ for $0 \leqq u \leqq c$ or $u \geqq c$ respectively [see (2.13)]. By the normalization 
condition (2.21), the factor $Z$ is determined:

$$
Z=R^{-1}\left(u, \lambda_{0}\right)
$$

Differentiating (3.17) with respect to $v$ and setting $v=1$, one gets

$$
\gamma\left(\Lambda\left(u, \lambda_{0}\right)\right)=\frac{u}{R\left(u, \lambda_{0}\right)} \frac{\partial R\left(u, \lambda_{0}\right)}{\partial u} .
$$

Using $\Lambda, \lambda_{0}$ as independent variables, this equation takes the form

$$
\begin{aligned}
\gamma(\Lambda) & =\frac{u}{R} \frac{\partial R}{\partial u}, \\
u & =u\left(\Lambda, \lambda_{0}\right)=\exp \left(\int_{\lambda_{0}}^{\Lambda} d z \beta^{-1}\right), \\
R & =R\left(u, \lambda_{0}\right)=R\left(u\left(\Lambda, \lambda_{0}\right), \lambda_{0}\right),
\end{aligned}
$$

with the overall dependence on $\lambda_{0}$ dropping out. Here $\lambda_{0}$ and $u$ are restricted by (2.13) such that

$$
\beta\left(\lambda_{0}\right) \neq 0, \quad R\left(u, \lambda_{0}\right) \neq 0 .
$$

Since $u$ is analytic in $\Lambda$, Eq. (3.21) may be continued to complex values of $\Lambda$. Thus $\gamma$ becomes an analytic function in the intersection $\mathscr{I}$ of $\mathscr{D}$ and the domain of $\Lambda$ for which $R$ is analytic in $u$ by (3.21). Here $\gamma$ is regular in $\mathscr{I}$ except at points $A=\lambda^{\prime}$ where one of the following conditions holds

$$
\begin{aligned}
& \beta\left(\lambda^{\prime}\right)=0, \\
& \left.\begin{array}{l}
R\left(u^{\prime}, \lambda_{0}\right)=0 \\
R\left(u^{\prime}, \lambda_{0}\right) \text { singular }
\end{array}\right\} \text { at } \quad u^{\prime}=\exp \left(\int_{\lambda_{0}}^{\lambda^{\prime}} d z \beta^{-1}\right) .
\end{aligned}
$$

The conditions (3.24) or (3.25) determine values $\lambda^{\prime}$ which are independent of $\lambda_{0}$. For a zero or singularity $u^{\prime}$ of $R$ depends on $\lambda_{0}$ according to

$$
u^{\prime}=c \exp \left(\int_{\lambda_{0}}^{\sigma} d z \beta^{-1}\right)
$$

where $\sigma$ is a fixed positive value of the coupling parameter [see (2.32)]. Hence the corresponding value of $\lambda^{\prime}$ depends only on $c, \sigma$, which are characteristic constants of the zero or singularity considered.

By its original definition, $\gamma$ is a real function in some real domain. Therefore the analytic continuations $\gamma(\lambda)$ and $\bar{\gamma}(\lambda)=\gamma^{*}\left(\lambda^{*}\right)$ coincide there and represent the same analytic function. Hence all complex singularities of $\gamma$ appear in conjugate pairs.

\section{Analyticity in the Momentum and Coupling Variables}

The analytic properties of $\beta$ established in the last section will now be used to set up the analytic continuation of $\Lambda$ in both variables $u$ and $\lambda$. To this end the identity (2.12)

$$
u=\exp \left(\int_{\lambda}^{1} d z \beta^{-1}\right)
$$


will be continued to complex values of $\Lambda, u$, and $\lambda$. A more explicit method expressing $\Lambda$ by (2.15) as a function of a single complex variable will be given below. The relation (4.1) was originally derived for real intervals (2.13) where $\beta$ does not vanish and stationary points of $\Lambda$ are absent. By deforming the path of integration, relation (4.1) may be continued analytically within the regularity domain $\mathscr{S}$ of $\beta^{-1}$. According to the results of the last section, $\mathscr{S}$ includes the image $\mathscr{D}$ of the regularity domain of $\Lambda$ in $u$ except for zeros of $\beta$. Thus

$$
u=u(\Lambda, \lambda)
$$

becomes an analytic function of $\Lambda$ and $\lambda$, regular for $\Lambda \in \mathscr{S}, \lambda \in \mathscr{S}$. The function $u$ may be singular at endpoint singularities where $\beta(\lambda)^{-1}$ and/or $\beta(\Lambda)^{-1}$ are singular. The quantity $\Lambda$ may be determined as a function of both complex variables $u$ and $\lambda$ by rewriting (4.1) in the form

$$
\exp \left(\int_{\lambda_{0}}^{A} d z \beta^{-1}\right)=\exp \left(\int_{\lambda_{0}}^{\lambda} d z \beta^{-1}\right) u
$$

and inverting the left hand side with respect to $\Lambda$. For $\lambda_{0}$, any regular point of $\beta^{-1}$ on the path of integration may be taken. For $\Lambda$ finite, $\beta^{-1}(\Lambda)$ regular and nonvanishing, the inversion is possible and $\Lambda$ becomes regular analytic in the variable

$$
v=\exp \left(\int_{\lambda_{0}}^{\lambda} d z \beta^{-1}\right) u
$$

In turn $v$ is a regular analytic function of $u$ and $\lambda$ except where $\beta^{-1}(\lambda)$ is singular. A singularity or zero of $\beta^{-1}(\lambda)$ may cause a singularity at the corresponding value $v^{\prime}$ of (4.4) leading to a singular surface

$$
\exp \left(\int_{\lambda_{0}}^{\lambda} d z \beta^{-1}\right) u=v^{\prime}
$$

of $\Lambda$ in the variables $u$ and $\lambda$. The constants $v^{\prime}$ are determined by

$$
v^{\prime}=\exp \left(\int_{\lambda_{0}}^{1} d z \beta^{-1}\right)
$$

where $\beta^{-1}(\Lambda)=0$ or singular. Likewise, a singularity of $\Lambda$ appears along (4.5) if

$$
\exp \left(\int_{\lambda_{0}}^{\infty} d z \beta^{-1}\right)=v^{\prime}
$$

converges along a path extended to infinity. In this case $\Lambda$ is unbounded near the singular surface.

It is of interest to apply this method to the Euclidean region. The relation (2.12) need not hold in this form if there are stationary points of $\Lambda$ for $u>0$. However, if the stationary points are restricted to a bounded region, the relation (2.12) can be continued from the original interval (2.13) to the infrared (ultraviolet) region. Then it holds for $0<u \leqq a$ as well as $b \lessgtr u$ with the appropriate branch of $A$ and suitable bounds $a, b$. In these regions, $\Lambda$ is a monotonic function of $u$ approaching limit 
values $\Lambda \rightarrow 0$ and $\Lambda \rightarrow \lambda_{\infty}$ (which may be infinite) for $u \rightarrow 0$ or $u \rightarrow \infty$. As a consequence, the integrals

$$
\int_{\lambda}^{0} d x \beta^{-1}, \quad \int_{\lambda}^{\lambda_{0}} d x \beta^{-1}
$$

must diverge at the upper limit. The zeros of $\beta$ at $\lambda=0$ and $\lambda=\lambda_{\infty}$ (if finite) are called strong zeros in contradistinction to weak zeros, where $\beta^{-1}$ is still integrable. As was shown in the last section, weak zeros appear for values of $\Lambda$ at stationary points $u^{\prime} \neq 0$.

On the other hand, if $\int_{\lambda}^{\infty} d x \beta^{-1}$ converges for the relevant branch of $\beta$, the effective coupling must be singular at the Euclidean momentum

$$
\frac{k^{2}}{\kappa^{2}}=\exp \left(\int_{\lambda}^{\infty} d x \beta^{-1}\right)
$$

with $\Lambda \rightarrow \infty$ when $k^{2}$ approaches this point. This generally does not imply the presence of tachyonic modes, it only means that the relation to an effective coupling defined by a vertex function is given by a singular transformation.

Equivalent information on the analytic properties of $\Lambda$ is obtained by continuing the functional relation (2.15) to complex values of $\lambda$ and $u$,

$$
\Lambda(u, \lambda)=\Lambda\left(\exp \left(\int_{\lambda_{0}}^{\lambda} d z \beta^{-1}\right) u, \lambda_{0}\right) .
$$

Here $\lambda_{0}$ denotes a fixed positive value below $\eta$. Equation (4.7) expresses $\Lambda$ as a function of the single variable (4.4). The regularity domain of $\Lambda$ in both variables $u$ and $\lambda$ can be described as follows. Let $\mathscr{R}\left(\lambda_{0}\right)$ be the region on the Riemann surface over the $v$-plane where $\Lambda\left(v, \lambda_{0}\right)$ is regular. Then $\Lambda(u, \lambda)$ is regular on the Riemann surface over the $u$-plane at any $u, \lambda$ with

$$
\exp \left(\int_{\lambda_{0}}^{\lambda} d z \beta^{-1}\right) u \in \mathscr{R}\left(\lambda_{0}\right), \quad \lambda \in \mathscr{S} .
$$

We will now use the analytic properties of $\gamma$, derived in the last section, in order to show that $R$ is analytic in both variables $u$ and $\lambda$. The differential equation (2.22) for $R$, with the normalization condition (2.21), can be solved by

$$
R(u, \lambda)=\exp \left(\int_{\lambda}^{\Lambda(u, \lambda)} d z \gamma \beta^{-1}\right)
$$

for values $u, \lambda$ from appropriate intervals (2.13). Since $\beta, \gamma$ are analytic and $\Lambda$ an analytic function of $u$ and $\lambda$, it follows that also $R$ is analytic in both variables. The actual domain of regularity is better described by continuing the relation (3.17) to complex values of $u$ and $\lambda$

$$
\begin{aligned}
& R(u, \lambda)=\exp \left(\int_{\lambda}^{\lambda_{0}} d z \gamma \beta^{-1}\right) R\left(v, \lambda_{0}\right), \\
& v=\exp \left(\int_{\lambda_{0}}^{\lambda} d z \beta^{-1}\right) u, \quad 0 \leqq \lambda_{0} \leqq \eta .
\end{aligned}
$$


The integration paths should avoid singularities of $\beta^{-1}$ and $\gamma$. According to this, $R(u, \lambda)$ is regular on the Riemann surface over the $u$-plane of any $u, \lambda$ with

$$
\exp \left(\int_{\lambda_{0}}^{\lambda} d z \beta^{-1}\right) u \in \mathscr{U}\left(\lambda_{0}\right), \quad \lambda \in \mathscr{T} .
$$

Here $\mathscr{U}\left(\lambda_{0}\right)$ denotes the region on the Riemann surface over the $v$-plane where $R\left(v, \lambda_{0}\right)$ is regular. $\mathscr{T}$ is the region where $\beta^{-1}$ and $\gamma \beta^{-1}$ are regular.

The propagators are real functions of Euclidean momenta in some real domain of the coupling parameter. For some definitions the effective coupling is real only in part of the Euclidean region. In these real domains of $u$ and $\lambda$ the analytic functions $\Lambda(u, \lambda)$ and $R(u, \lambda)$ coincide with their complex conjugates $\Lambda^{*}\left(u^{*} \lambda^{*}\right)$ or $R^{*}\left(u^{*}, \lambda^{*}\right)$ respectively; hence they represent the same analytic functions.

We conclude with some general statements on the singularities of the effective coupling and propagators. For given $\lambda_{0}$, some of the singularities of $\Lambda\left(v, \lambda_{0}\right)$ or $R\left(v, \lambda_{0}\right)$ with respect to $v$ are given by physical requirements such as particle poles, thresholds, resonances, etc. Other singularities arise from zeros of the propagators involved in the definition of $\Lambda$. If a singularity of $\Lambda\left(v, \lambda_{0}\right)$ or $R\left(v, \lambda_{0}\right)$ is located at $v=\mu^{2}\left(\lambda_{0}\right)$, the position of the corresponding singularity of $\Lambda(u, \lambda)$ or $R(u, \lambda)$ is at

$$
u=\mu^{2}\left(\lambda_{0}\right) \exp \left(\int_{\lambda}^{\lambda_{0}} d z \beta^{-1}\right)=\mu^{2}(\lambda) .
$$

This is the generalization of (2.28) to the dependence of a singular point on the complex-valued coupling constant. The position of a pole or other singularity where $\Lambda$ becomes infinite is

$$
u=\mu^{2}(\lambda)=\exp \left(\int_{\lambda}^{\infty} d z \beta^{-1}\right),
$$

as follows from (4.1) (with suitable path of integration).

As a consequence of the relations (4.8) and (4.11), singularities of the effective coupling and the propagators may only occur

(i) at $k^{2}=0$ or $k^{2}=\infty$;

(ii) at fixed values $\lambda=\lambda^{\prime}$ where $\beta^{-1}$ is singular or, in case of a propagator, where $\gamma \beta^{-1}$ is singular;

(iii) along surfaces

$$
\exp \left(\int_{\lambda_{0}}^{\lambda} d z \beta^{-1}\right) u=\mu^{2}\left(\lambda_{0}\right)
$$

provided there is a singularity at $u=\mu^{2}\left(\lambda_{0}\right)$ for $\lambda=\lambda_{0}$.

Acknowledgements. This work has been supported in part by the National Science Foundation (R.O) and by the Federal Republic of Germany (W.Z.).

\section{References}

1. Eckmann, J.-P., Magnen, J., Sénéor, R.: Commun. Math. Phys. 39, 251-271 (1975)

2. Glimm, J., Jaffe, A., Spencer, T.: The cluster expansion in constructive quantum field theory. In: Lecture Notes in Physics, Vol. 25. Berlin, Heidelberg, New York: Springer 1973

3. Khuri, N.N. : Phys. Rev. D 12, 2298-2310 (1975) 
4. t' Hooft, G.: Can we make sense out of quantum Chromodynamics? In: The whys of subnuclear physics (Erice 1977), pp. 943-971. New York: Plenum Press 1979

5. Oehme, R.: Phys. Lett. 71 B, 169-171 (1977)

6. Oehme, R., Zimmermann, W.: Phys. Lett. 79B, 314-316 (1978)

7. Oehme, R., Zimmermann, W.: Phys. Lett. 100B, 273-275 (1981)

8. Gross, D.: 1975 Les Houches Lectures. In: Methods in Field Theory, pp. 162-191. Amsterdam: North-Holland 1976

9. Ovrut, B., Schnitzer, H.: Low energy and threshold calculations using effective field theory. Nucl. Phys. B (to be published)

10. Oehme, R., Zimmermann, W.: Phys. Lett. 79B, 93-96 (1978)

11. Popović, D. : Prog. Theor. Phys. 58, 300-315 (1977)

12. Callan, C., Gross, D.: Private communication

13. Kogut, J.: Progress in lattice gauge theory, Santa Barbara Report NSF-ITP-80-16

14. Gross, D.: A review of the semiclassical approach to QCD, Princeton Report, 1980

15. Khuri, N. : Phys. Rev. D 23, 2285 (1981)

16. Epstein, H., Iliopoulos, J.: The arbitrariness in the perturbation series in non-abelian gauge theories (preprint) Laboratoire de Physique Theórique de l'Ecole Normale Superieure, 1981

17. Khuri, N., McBryan, O.: Phys. Rev. D 20, 881-886 (1979)

18. Adler, S.: Phys. Rev. D 23, 2905 (1981)

19. Frishman, Y., Horsley, R., Wolff, U.: Phys. Lett. 96 B, 302 (1980)

20. Curci, G., Ferrari, R.: Nuovo Cimento 35 A, 273-279 (1976); Phys. Lett. 63B, 91-93 (1976)

21. Kugo, T., Ojima, I. : Phys. Lett. 73B, 459-461 (1978); Prog. Theor. Phys. 60, 1869-1889 (1978); 61, 294-314 (1979); Prog. Theor. Phys. Suppl. No. 66 (1979)

22. Oehme, R., Zimmermann, W.: Phys. Rev. D21, 1661-1671 (1980)

Communicated by R. Stora

Received June 30, 1981 
